\title{
International Spinal Research Trust Clinical Initiative
}

Various strategies for repairing spinal cord injuries in experimental animals have recently succeeded in inducing significant amounts of axon regeneration with return of function. These studies point the way towards the first arrivals of treatment in human patients. ISRT wishes to put in place the assessment strategies that will be needed to determine the results of human spinal cord repair treatments.

Treatment of spinal cord injury will almost certainly involve implantation of cells into the lesion site, and to this will be added infusions of growth factors and blockers of inhibitory molecules. Because of the necessity for direct intervention at the lesion site it is essential that the first treatments are delivered to a region of the cord where any collateral damage from the surgery will not have significant adverse effects on the patient. This makes it unlikely that the first treatments could be delivered to patients with cervical cord lesions. ISRT considers that the most favourable group of patients for a safe trial of the first treatments will be those with functionally complete lesions in the lower part of the thoracic cord.

Axon treatment in spinal cord injuries in animal models has been induced for up to $3 \mathrm{~cm}$. It may be that regeneration over longer distances will be seen in larger animals, but it is necessary to plan on the basis that the first treatments in humans will produce axon regeneration over this distance. This amount of regeneration would produce readily detectable functional changes in the cervical cord, but functional effects will be more difficult to assess in thoracic cord.

ISRT wishes to fund studies that will lead to the development of techniques for detecting functional, physiological and structural changes over two or three spinal segments following spinal cord repairs, and for high resolution imaging of the progress of lesions and the behaviour of implanted cells. Assessment techniques with the necessary resolution are not presently available in routine clinical practice, and their development will therefore require collaboration between clinical departments and clinical or basic science departments. These techniques will initially be directed towards thoracic lesions, but it is important that they also be applicable to lesions in other parts of the cord. In order to develop the necessary techniques ISRT wishes to fund longitudinal studies of recently injured patients, which focus on detailed functional, physiological and structural events in the intermediate vicinity of the lesion.

ISRT invites applications from multidisciplinary teams. Teams should include one or more spinal injury centres in the UK, sensory and motor physiology, autonomic physiology and imaging. Bladder and bowel physiology may also be relevant. The anticipated level of funding is four or possibly five salaries with costs, and the duration three years in the first instance.

The application procedures is in two steps. In the first instance ISRT invites letters of intent with a maximum length of four sides of A4. The letter should detail the logistics of the study, with plans for access to patients and for their treatment and physiotherapy. The assessment techniques that will be studied should be described and their rationale explained. The specialties of staff employed on the study, the places where they will work and the plans for their supervision should be detailed. A provisional rough estimate of budget should be attached. ISRT will assess the preliminary applications, and may invite detailed proposals immediately, or may enter into dialogue with applicants to refine the letters of intent before inviting detailed applications.

For application forms, and to discuss details contact Peter Banyard or John Cavanagh at International Spinal Research Trust, Tel: 01483 898786; fax: 01483898763 